\title{
A Novel Compact Low Loss Waveguide Image Rejection Filter Based on a Backward Coupler with Band Pass Filters for $100 \mathrm{GHz}$ Band
}

\author{
Shin'ichiro Asayama • Yutaka Hasegawa • \\ Akira Mizuno · Hideo Ogawa · Toshikazu Onishi
}

Received: 1 November 2014 / Accepted: 6 February 2015 /

Published online: 25 February 2015

(C) The Author(s) 2015. This article is published with open access at Springerlink.com

\begin{abstract}
This paper describes the design and test observation result of a novel waveguide image rejection filter (IRF). The IRF is based on a quadrature hybrid coupler as a backward coupling structure, followed by Band Pass Filters (BPFs), and matched loads for the image-frequency termination. A prototype IRF shows return loss of better than $18 \mathrm{~dB}$, and an image rejection ratio of more than $25 \mathrm{~dB}$ over $4 \mathrm{GHz}$ band for stratospheric ozone spectra at $110 \mathrm{GHz}$ when the LO frequency and IF frequency are $104 \mathrm{GHz}$ and $6 \mathrm{GHz}$, respectively. We installed the prototype IRF into an ozone-measuring system and successfully observed an ozone spectrum at $110 \mathrm{GHz}$ in single sideband (SSB) mode. This IRF has the advantages of low transmission loss, compact size, and easy scalability for sub-millimeter frequencies.
\end{abstract}

Keywords Waveguide - Backward coupler - Image rejection filter ·

Millimeter wave $\cdot$ Ozone spectrum

\footnotetext{
S. Asayama $(\square)$

NAOJ Chile Observatory, National Astronomical Observatory of Japan,

2-21-1 Osawa, Mitaka, Tokyo, 181-8588, Japan

e-mail: shinichiro.asayama@nao.ac.jp

Y. Hasegawa · H. Ogawa · T. Onishi

Department of Physical Science, Graduate School of Science, Osaka Prefecture University,

1-1 Gakuen-cho, Naka-ku, Sakai, Osaka, 599-8531, Japan
}

A. Mizuno

Solar-Terrestrial Environment Laboratory, Nagoya University,

Furo-cho, Chikusa-ku, Nagoya, Aichi, 464-8601, Japan 


\section{Introduction}

There is a strong demand to use single sideband (SSB) or sideband separating (2SB) receivers for spectral line observations in astronomical and remote sensing applications at millimeter/sub-millimeter wavelengths. In this decade, waveguide-type 2SB superconductor-insulator-superconductor (SIS) mixers have been developed [1-7], and adopted for the receiver bands of the Atacama large millimeter/sub-millimeter array (ALMA) [8].

For the 2SB mixer, the image rejection ratio (IRR), which is the value of rejecting power from the image sideband to the signal sideband in the heterodyne mixer receiver in SSB mode, depends on the quality of SIS devices and is changed by tuning the SIS bias voltage settings since the amplitude imbalance between the two SIS mixers will deteriorate the image rejection ratio [9]. However, a stable image rejection ratio is quite important for the accuracy of intensity calibration of molecular line observations, especially during long-term monitoring applications.

In this paper we report a novel waveguide Image Rejection Filter (IRF) based on a backward coupling structure. Waveguide backward coupling structures have been used for waveguide Orthomode Transducers at millimeter/sub-millimeter wavelengths, and have demonstrated excellent performance [10, 11]. The IRF is based on a quadrature hybrid coupler as a backward coupling structure, followed by Band Pass Filters (BPFs), and matched loads for the image-frequency termination. The advantage of this approach is that the IRF can be fabricated with a small waveguide structure and low transmission loss, and, in addition, a stable and reliable image rejection ratio can be achieved. Even though this scheme is fixed tuned, this is quite practical for some applications with no frequency change required. To demonstrate the feasibility of the IRF, we fabricated a prototype IRF and installed it into an ozone-measuring system with an SIS mixer and successfully observed an ozone spectrum at $110 \mathrm{GHz}$ in SSB mode.

\section{Filter Details}

\subsection{IRF Based on a Backward Coupler with Band Pass Filters}

Figure 1 depicts the block diagram of a waveguide IRF based on a backward coupler with Band Pass Filters (BPFs). It has a similar layout and uses the same concept of a hybrid-coupled multiplexer [12]. The IRF is composed of a quadrature hybrid coupler, followed by BPFs and matched loads. Figure 1a shows the case of input signals with frequencies outside the BPF pass-band. The input signal at port 1 is divided at $-3 \mathrm{~dB}$ with a 90-degree phase difference between the hybrid coupler through port 3 and 4 . The two signals are reflected by the BPFs, and sent backward and recombined in-phase at port 2 and out-of-phase at port 1 (cancel out). The net effect is that the input signal at port 1 is fully coupled to port 2 . Figure $1 \mathrm{~b}$ shows the case of input signals with frequencies within the BPF pass-band. The two signals at port 3 and 4 are allowed to pass through the BPFs, and are terminated to the matched loads. With the BPF pass-band set to the image band frequencies, this network can be used as a IRF. 


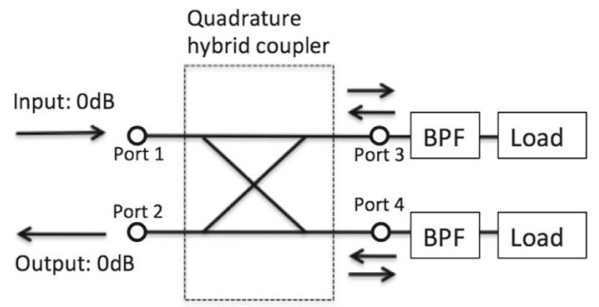

(a) Signals with frequencies outside the BPF pass-band

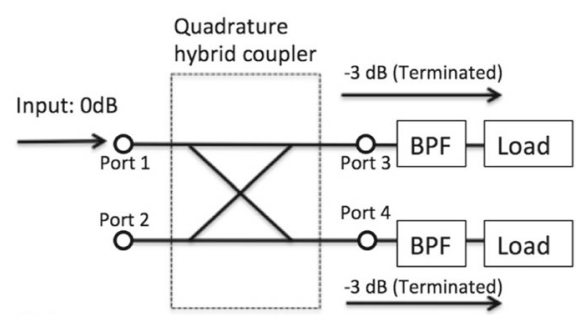

(b) Signals with frequencies within the BPF pass-band

Fig. 1 Block diagram of a backward coupling structure consisting of a quadrature hybrid coupler, followed by BPF with matched loads. a Signal with frequencies within the BPF pass-band, b Signal with frequencies outside the BPF pass-band

\subsection{Filter Design}

A three-dimensional view of the waveguide structure is illustrated in Fig. 2. It contains a seven-section E-plane branch line coupler (BLC) used as the quadrature hybrid coupler, followed by two five-section H-plane BPFs with round chamfer, as in Fig. 1. The waveguide size is WR-10 $(2.54 \times 1.27 \mathrm{~mm})$ for the stratospheric ozone spectra at $110 \mathrm{GHz}$. To optimize the design, we used a commercial 3D electromagnetic simulation software (HFSS ${ }^{\mathrm{TM}}$, Ansoft Corporation). The structure can be constructed in two mechanical blocks using conventional E-plane split-block techniques.

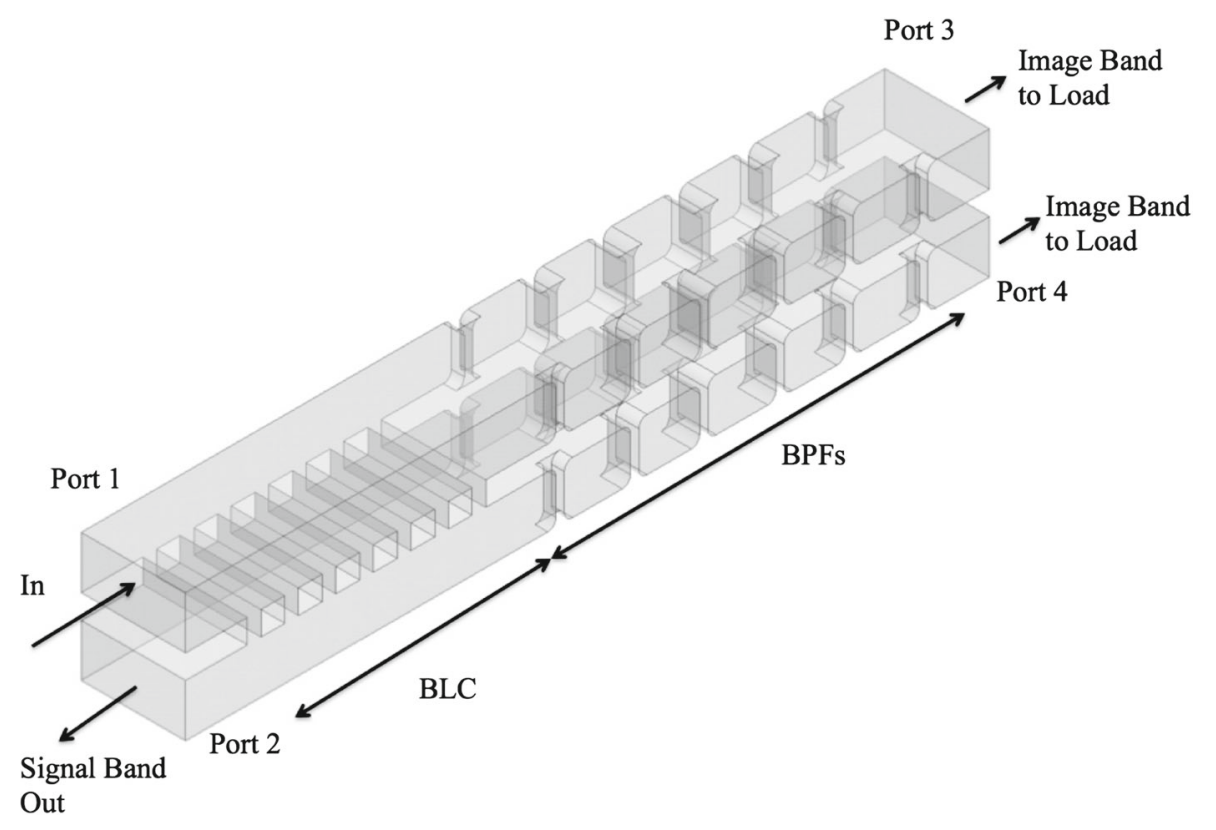

Fig. 2 Waveguide structure of the IRF (BLC and BPFs) 


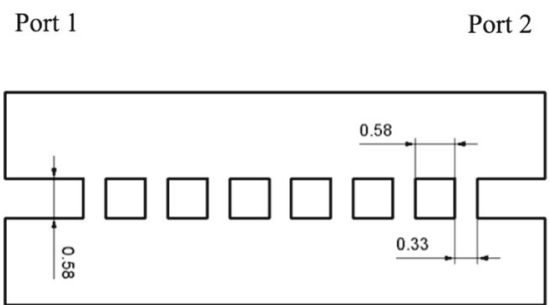

Port 4

Port 3
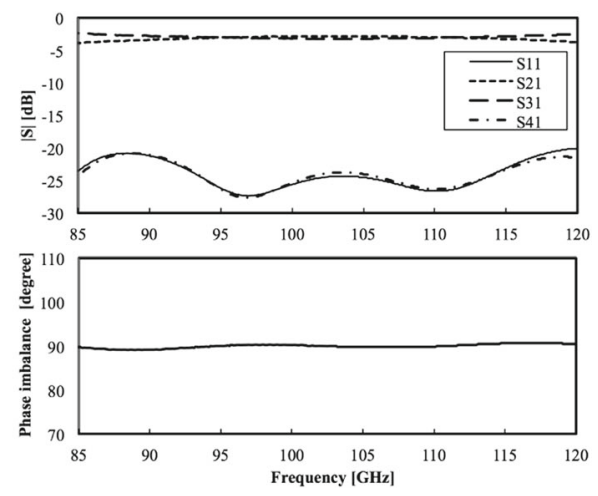

Fig. 3 Left: Diagram with dimensions (in mm) of the BLC of Fig. 2. Right: The simulated of the BLC. Top plot shows the input return loss (S11), isolation (S41), and coupling to the main and side waveguides (S21 and S31, respectively), bottom plot shows the phase imbalance

Waveguide E-plane BLCs have been studied and proven to be suitable for millimeter and terahertz-wave applications such as radio astronomy instrumentation [13-15]. To simplify the design and fabrication, we have kept the main guides at the full height, and made all the branch lines the same height, with equal length and spacing. The BLC dimensions are shown in Fig. 3 (Left). Each shunt is $0.33 \mathrm{~mm}$ wide and $1.27 \mathrm{~mm}$ deep. The centers of the slots are spaced by $0.58 \mathrm{~mm}$. The simulated results of the BLC are shown in Fig. 3 (Right). E-plane split-block waveguide BPFs have been extensively studied for millimeter and sub-millimeter applications (see e.g. [16-18]). The BPF dimensions are shown in left top and bottom of Fig. 4. The waveguide irises have the thickness of $0.2 \mathrm{~mm}$ to provide adequate mechanical strength, and have round corners (radius $0.2 \mathrm{~mm}$ ) to allow machining with an endmill. We have optimized the the cavity lengths and iris openings to achieve the return loss of greater than $30 \mathrm{~dB}$ within $96-100 \mathrm{GHz}$ for the good image rejection performance. The BPF simulated responses are shown in Fig. 4 (Right).

The entire four-port structure in Fig. 2 has been simulated without taking into account the effects of ohmic losses (only perfect conductors were considered). The
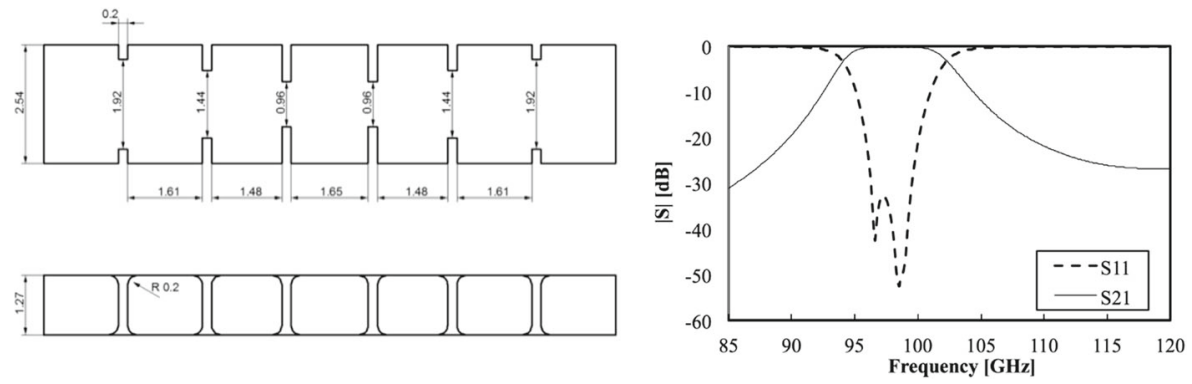

Fig. 4 Diagram with dimensions (in mm) of the the BPF of Fig. 4 showing H-plane cut (Left Top) and E-plane cut (Left bottom). Right: Simulated performance of the BPF showing the input return loss (S11), and transmission (S21) 


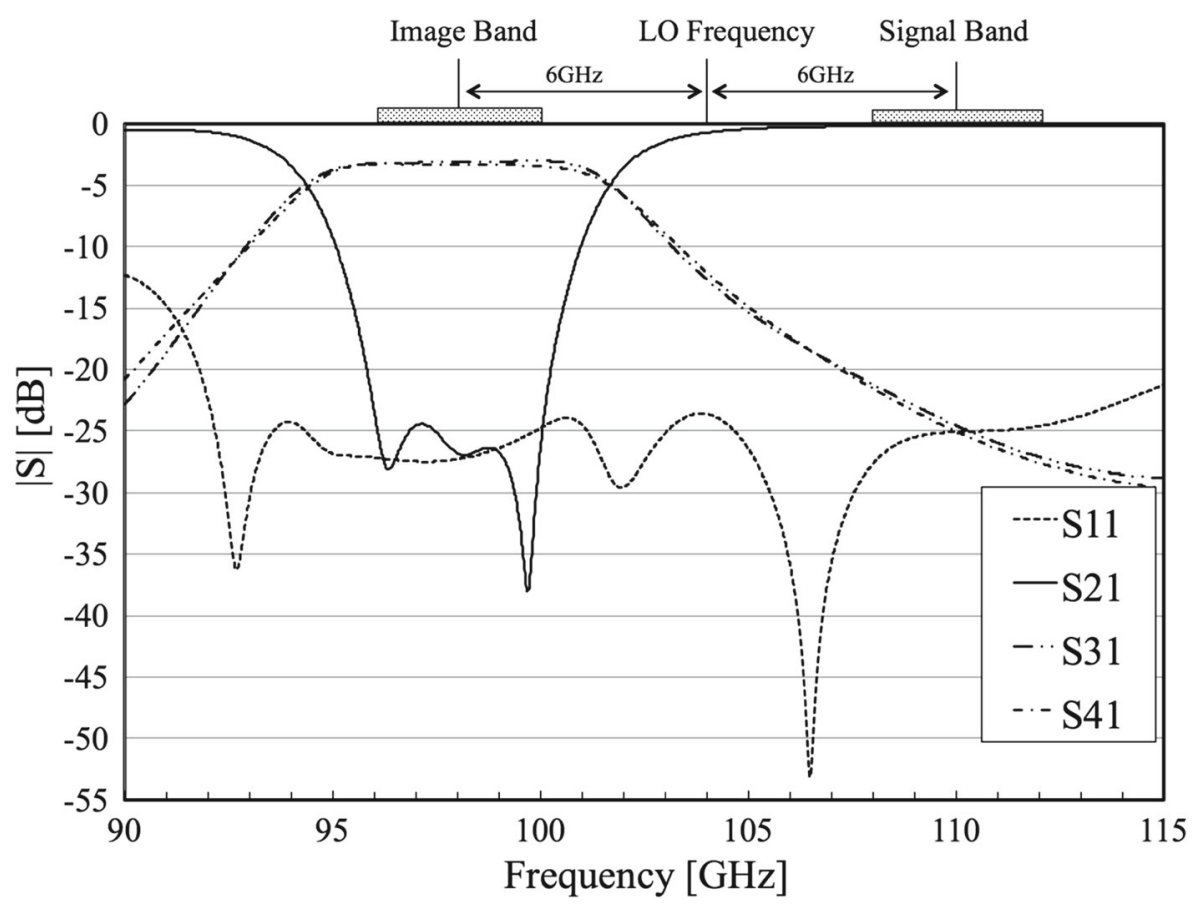

Fig. 5 Simulated performance of the IFR showing the input return loss (S11), transmission (S21) and coupling to the termination ports of port 3 and 4 (S13 and S14, respectively)

simulated results are shown in Fig. 5. With this waveguide structure, we can achieve an image rejection ratio of more than $25 \mathrm{~dB}$ over $4 \mathrm{GHz}$ band for stratospheric ozone spectra at $110 \mathrm{GHz}$ when the LO frequency and IF frequency are $104 \mathrm{GHz}$ and $6 \mathrm{GHz}$, respectively. From the electromagnetic simulations, we confirmed that mechanical errors of $20 \mu \mathrm{m}$ have little impact on the reflection and transmission coefficients, and can only cause a BPF center frequency shift of less than $1.6 \%$ on the IRF performance.

\subsection{Fabrication and Measurements}

The split-block waveguide IRF assembly for the filter is illustrated in Fig. 6. The IRF was manufactured out of 6061 aluminum alloy. This material has good electrical conductivity and resistance to environmental degradation, thus eliminating the need for gold plating. This material also has good thermal conductivity, which is beneficial for a cooled receiver with an SIS mixer. A photograph of the two halves of the waveguide block is shown in Fig. 7. The overall dimension of the IRF is $20 \times 24 \times 48 \mathrm{~mm}$.

The performance of the prototype image rejection filter was measured using a Millimeter-wave Vector Network Analyzer (Agilent E8361C PNA with OML WR-10 VNA Extension module) at National Institute of Information and Communications 


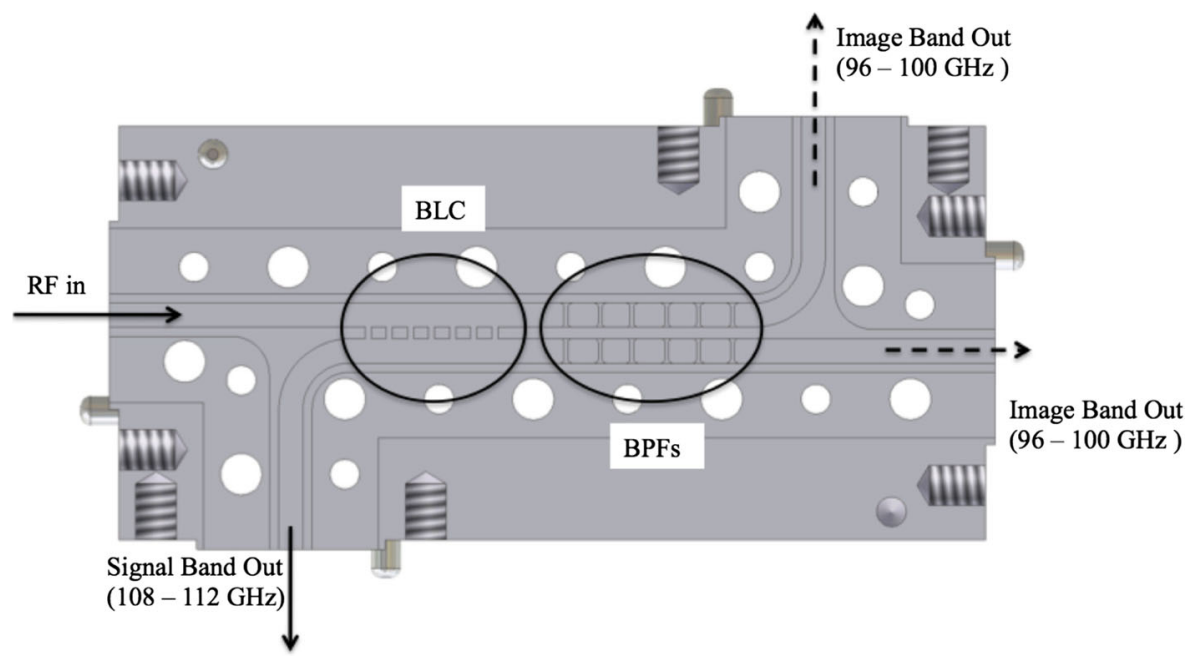

Fig. 6 Configuration of the IRF for split block

Technology (NICT) in Japan. The network analyzer was calibrated with TRL (Through-Reflect-Line) calibration standards. Image band output ports of the IRF were terminated with waveguide terminations. Figure 8 shows simulated and measured results of the IRF. Overall, we found that the measurements closely follow the simulation results. The BPF resonant frequency shifts by only $0.4 \mathrm{GHz}(0.4 \%)$. The small difference between the simulation results and the measurement results can be explained by the fabrication error of $\sim 5 \mu \mathrm{m}$ at the BPF section. The average measured transmission loss is less than $0.3 \mathrm{~dB}$ at the target signal band of $110 \mathrm{GHz}$. The microwave insertion loss of aluminum is expected to decrease by a factor of $\sim 2$ when it is cooled from room temperature to $4 \mathrm{~K}$ [19]. Since the IRF will be operated at $4 \mathrm{~K}$ in front of an SIS mixer, we expect a maximum insertion loss of below $0.15 \mathrm{~dB}$ under operating conditions. The measured return loss is more than $18 \mathrm{~dB}$ in the target
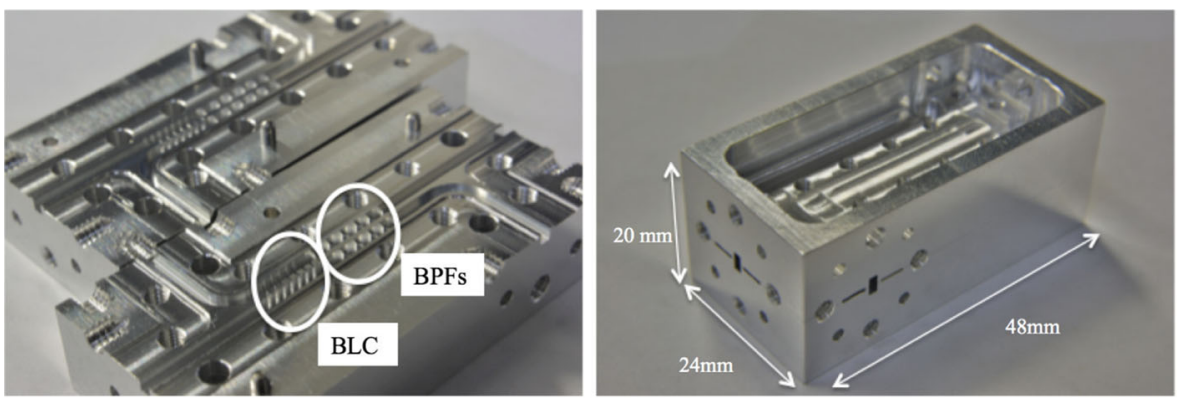

Fig. 7 Left: The two halves of the split-block, showing the internal waveguide circuitry. Right: Assembled IRF showing the input and output waveguides 

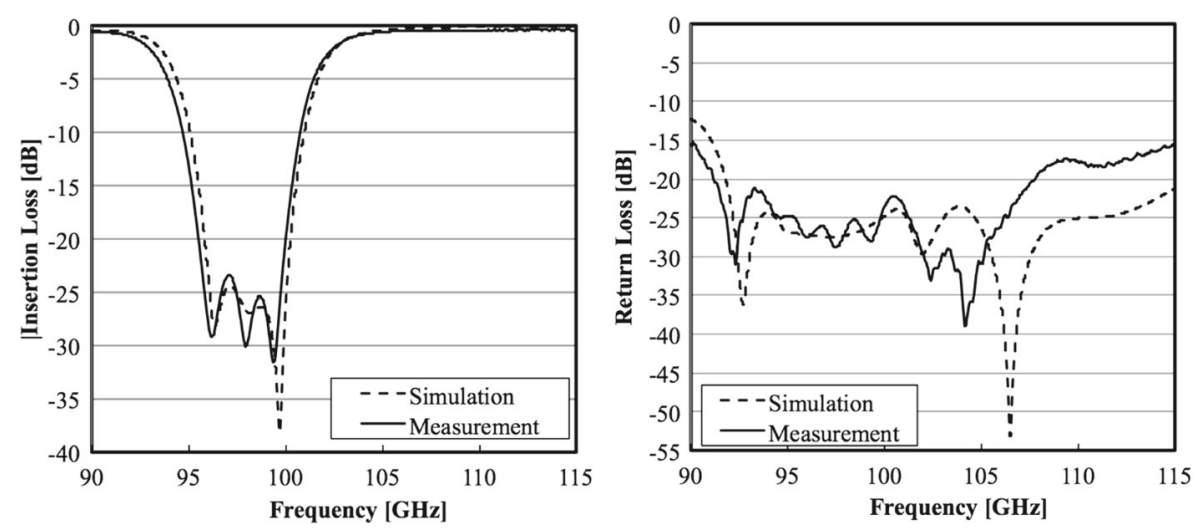

Fig. 8 Measured (solid lines) and simulated (dashed lines) transmission loss (left panel) and Input Return Loss (right panel) of the prototype IRF

signal band of $110 \mathrm{GHz}$. The discrepancy between simulated and measured return loss is believed to be caused by the mismatch at the input port. The image signal suppression is more than $25 \mathrm{~dB}$ in the image band of $98 \mathrm{GHz}$ when the LO frequency and IF frequency are $104 \mathrm{GHz}$ and $6 \mathrm{GHz}$, respectively.

\section{Results}

\subsection{Receiver Performance}

The prototype IRF was installed into an SIS mixer test system. The block diagram of the measuring system is shown in Fig. 9. The double sideband (DSB) SIS mixer adopted here was developed at Osaka Prefecture University [20]. The IRF was inserted between the feed horn and the SIS mixer, then image band signals were terminated to $4 \mathrm{~K}$ termination loads. The receiver components were mounted on the $4 \mathrm{~K}$ cold stage of a test dewar, and kept at a temperature of around $4 \mathrm{~K}$ by a closed-cycle refrigerator. A kapton film of thickness $25 \mu \mathrm{m}$ and a GoreTex membrane of thickness $0.5 \mathrm{~mm}$ were used as a vacuum window and Infrared (IR) filter, respectively. RF and LO signals were coupled to the SIS mixer using a waveguide directional coupler with a coupling efficiency of $-25 \mathrm{~dB}$. The IF output from the mixer was first amplified by a cooled High-Electron-Mobility Transistor (HEMT) amplifier associated with a cooled isolator then further amplified at room temperature. The noise temperatures of the receiver were measured by a standard Y-factor method. The DSB receiver noise temperature at the LO frequency $104 \mathrm{GHz}$ with $4-8 \mathrm{GHz}$ IF was about $20 \mathrm{~K}$. The SSB noise temperature with the IRF was about $40 \mathrm{~K}$. In a standard Y-factor method, the receiver noise temperature in the SSB system is just twice of that observed in the DSB system. Therefore, there was no clear system sensitivity degradation due to the IRF. With the installation of the IRF, no IF output power ripple was observed in 4-8 GHz IF. This means that the image band was well terminated to the termination loads. To 


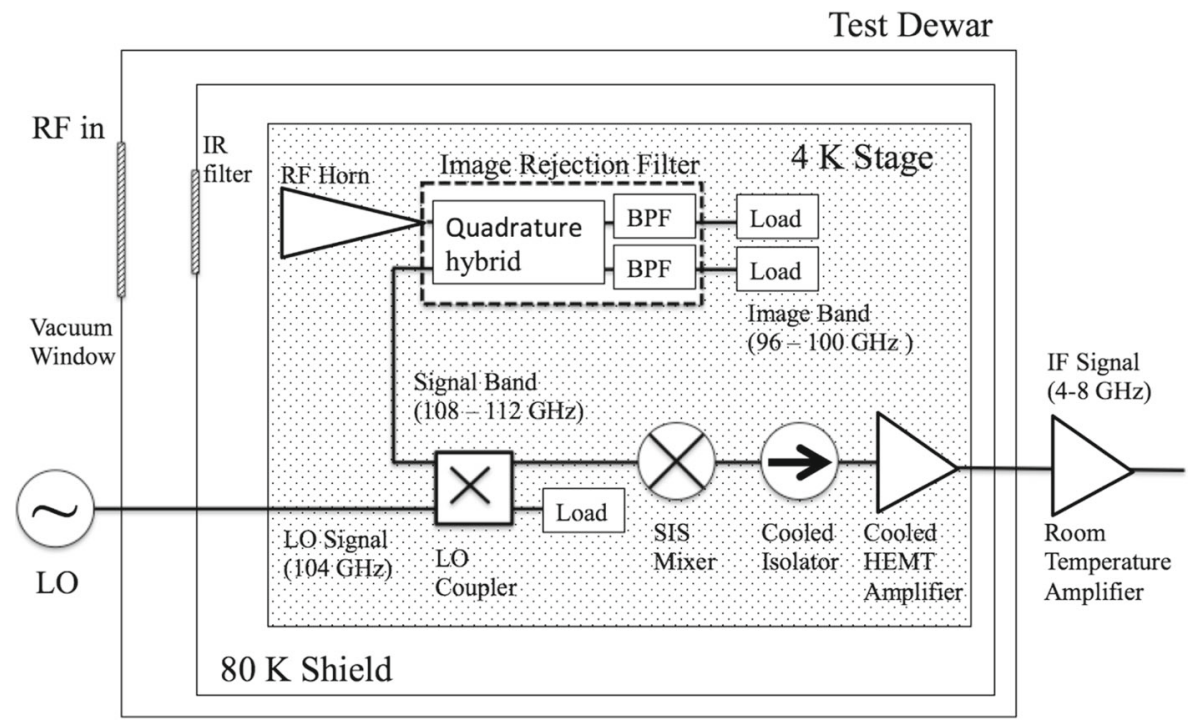

Fig. 9 Block diagram of the measurement setup for the IRF with an SIS mixer

measure the image rejection ratio, $\mathrm{CW}$ (continuous wave) signals were injected into the upper and lower sidebands of the DSB mode (without the IRF) and the SSB mode (with the IRF), then the image rejection ratio was calculated by comparing the downconverted signals at the IF band. The measured image rejection ratio was more than $25 \mathrm{~dB}$ at $110 \mathrm{GHz}$ when the LO frequency and IF frequency are $104 \mathrm{GHz}$ and $6 \mathrm{GHz}$, respectively. This result was consistent with the VNA measurements, as expected.

\subsection{Ozone Observation}

A millimeter-wave radiometer was installed at Rikubetsu, Japan $\left(43.5^{\circ} \mathrm{N}, 143.8^{\circ} \mathrm{E}\right)$ in March 1999, to monitor the vertical distribution of ozone and temporal ozone

Fig. 10 Atmospheric ozone spectra obtained in the DSB (without the IRF) and SSB (with the IRF) modes

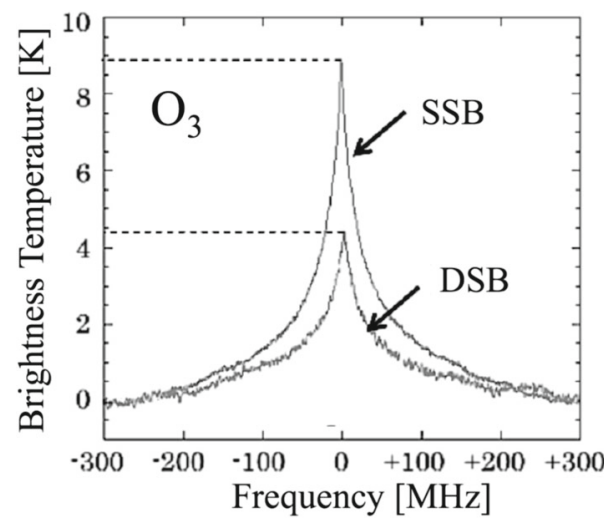


variations in the stratosphere. Since November 1999, vertical profiles of the ozonemixing ratio in the altitude range from 22 to $60 \mathrm{~km}$, with measurements at twokilometer altitude intervals have been monitored [21]. We installed the prototype IRF into the atmospheric ozone-measuring system in August 2013. The observed atmospheric ozone spectra are shown in Fig. 10. These spectra are obtained in DSB mode (without the IRF) and SSB mode (with the IRF). There was no clear system sensitivity degradation due to the IRF. The temperature difference between SSB and DSB is due to the calibrating method. This measuring system calibrates the brightness temperature with the assumption that the receiver is in the SSB mode. Therefore, the brightness temperature of the ozone spectrum observed in the DSB mode is just half that observed in the SSB mode, as expected. These results thus indicate that good image rejection is achieved using the waveguide BSF.

Since the IRF is a passive waveguide component, the IRR does not depend on the DSB SIS mixer. Therefore, this IRF has the advantages of stable operation, and accuracy of intensity calibration of the atmospheric ozone spectra, especially during long-term monitoring applications. The IRF has demonstrated reliable and stable operation in more than a year since August 2013.

\section{Conclusion}

A $100 \mathrm{GHz}$ band novel waveguide IRF was designed, fabricated, and tested. The experimental results agree well with the simulation results. The measured room temperature insertion loss was less than $0.4 \mathrm{~dB}$, the reflection was less than $18 \mathrm{~dB}$, image rejection ratio of more than $25 \mathrm{~dB}$ over $4 \mathrm{GHz}$ band for stratospheric ozone spectra at $110 \mathrm{GHz}$ when the LO frequency and IF frequency are $104 \mathrm{GHz}$ and $6 \mathrm{GHz}$, respectively. We installed the IRF into an ozone-measuring system and successfully observed an ozone spectrum at $110 \mathrm{GHz}$ in SSB mode. The IRF has demonstrated reliable and stable operation in more than a year. The IRF has the advantage of compact size, and it can be scaled to submillimeter frequencies.

Acknowledgments Authors would like to thank K. Suzuki (Nagoya Univ.) for high-precision machining of the IRF. We are grateful to A. Kasamatsu, K. Kikuchi and S. Otiai (National Institute of Information and Communications Technology) for measuring the IRF with VNA. This work was financially supported in part by a Grant-in-Aid for Scientific Research from the Ministry of Education, Culture, Sports, Science and Technology of Japan (No. 22244014, and 26247026). This work was also supported by JST/JICA (Japan Science and Technology Agency/Japan International Cooperation Agency), SATREPS (Science and Technology Research Partnership for Sustainable Development). Y. Hasegawa was supported by JSPS Research Fellowship for Young Scientists DC1 (No. 26-12320).

Open Access This article is distributed under the terms of the Creative Commons Attribution License which permits any use, distribution, and reproduction in any medium, provided the original author(s) and the source are credited. 


\section{References}

1. S. Asayama, H. Ogawa, T. Noguchi, K. Suzuki, H. Andoh, and A. Mizuno, An integrated sidebandseparating SIS mixer based on waveguide split block for $100 \mathrm{GHz}$ band with 4.0-8.0 GHz IF, Int. J. Infrared Millim. Waves, 25(1), 107-117 (2004).

2. A. R. Kerr, S.-K. Pan, S. M. X. Claude, P. Dindo, A. W. Lichtenberger, and E. F. Lauria, Development of the ALMA-North America Sideband-Separating SIS Mixers, Microwave Symposium Digest (IMS), IEEE MTT-S International, 1-4, (2013).

3. B. Billade, V. Belitsky, A. Pavolotsky, I. Lapkin, and J. Kooi, Alma band 5 (163-211 ghz) sideband separation mixer, Proc. 20th Int. Symp. on Space THz Tech., 19-23 (2009).

4. S. M. X., Claude, Sideband-Separating SIS Mixer for ALMA Band 7, 275-370 GHz, Proc. 14th Int. Symp. on Space THz Tech., 22-24 (2003).

5. M. Kamikura, Y. Tomimura, Y. Sekimoto, S. Asayama, W. L. Shan, N. Satou, Y. Iizuka, T. Ito, T. Kamba, Y. Serizawa, and T. Noguchi, A 385-500 GHz sideband-separating (2SB) SIS mixer based on a waveguide split-block coupler, Int. J. Infrared and Millim. Waves, 27(1), 37-53 (2006).

6. F. P. Mena, J. W. Kooi, A. M. Baryshev, C. F. J. Lodewijk, R. Hesper, G. Gerlofsma, T. M. Klapwijk, and W. Wild, A Sideband-separating Heterodyne Receiver for the 600-720 GHz Band, IEEE Trans. Microwave Theory Tech, 59(1), 166-177 (2011).

7. D. Maier, J. Reverdy, D. Billon-Pierron, A. Barbier, Upgrade of EMIR's Band 3 and Band 4 Mixers for the IRAM 30 m Telescope, IEEE Trans. Terahertz Sci. Tech, 2(2), 215-221 (2012).

8. A. Wootten and A. R. Thompson, The Atacama Large Millimeter/Submillimeter Array, Proc. IEEE, 97(8), 1463-1471, (2009).

9. S. M. X. Claude, C. T. Cunningham, A. R. Kerr, and S.-K. Pan, Design of a Sideband-Separating Balanced SIS Mixer Based on Waveguide Hybrids, ALMA Memo 316 (2000).

10. A. Navarrini, and R. Nesti, Symmetric reverse-coupling waveguide orthomode transducer for the 3mm band, IEEE Trans. IEEE Trans. Microwave Theory Tech., 57(1), 80-88 (2009).

11. A. Navarrini, C. Groppi, and G. Chattopadhyay, A waveguide orthomode transducer for 385-500 GHz, Proc. 21th Int. Symp. on Space THz Tech., 23-25 (2010).

12. R. J. Cameron and M. Yu, Design of manifold-coupled multiplexers, IEEE Microwave Magazine, 8(5), 46-59, (2007).

13. H. Andoh, S. Asayama, H. Ogawa, N. Mizuno, A. Mizuno, T. Tsukamoto, T. Sugiura and Y. Fukui, Numerical Matrix Analysis for Performances of wideband $100 \mathrm{GHz}$ Branch-line Couplers, Int. J. Infrared Millim. Waves, 24(5), 773-788 (2003).

14. S. Srikanth and A. Kerr, Waveguide Quadrature Hybrids for ALMA Receivers, ALMA Memo 343 (2001).

15. H. Rashid, D. Meledin, V. Desmaris, and V. Belitsky, Novel Waveguide 3 dB Hybrid With Improved Amplitude Imbalance, IEEE Microwave and Wireless Components Letters, 24(4), 212-214 (2014).

16. B. Yang, Z.-P. Li, J. Zhang, X. Yao, C. Zheng, X. Shang, and J. Miao, Design of H-Plane Inductance Diaphragm Waveguide Band-Pass Filter for Millimeter Imaging Frontend, Progress In Electromagnetics Research C 48, 141-150 (2014).

17. V. Furtula and M. Salewski, W-band waveguide bandpass filter with E-plane cut, Review of Scientific Instruments 85(7), 074703 (2014).

18. V. Furtula, H. Zirath, H and M. Salewski, Waveguide Bandpass Filters for Millimeter-Wave Radiometers, Int. J. Infrared and Millimeter Waves, 34(12), 824-836 (2013).

19. R. Finger, and A. R. Kerr, Microwave loss reduction in cryogenically cooled conductors, Int. J. Infrared Millim. Waves, 29(10), 924-932 (2008).

20. S. Asayama, T. Noguchi, and H. Ogawa, A fixed-tuned W-band waveguide SIS mixer with 4.0-7.5 GHz IF, Int. J. Infrared and Millimeter Waves, 24(7), 1091-1099 (2003).

21. T. Nagahama, H. Nakane, H., Y. Fujinuma, A. Morihira, A. Mizuno, H. Ogawa, and Y. Fukui, Groundbased millimeter-wave radiometer for measuring the stratospheric ozone over Rikubetsu, Japan, J. of the Meteorological Society of Japan 85(4), 495-509 (2007). 